\title{
Prevalence of Malignancy Among Urban Black Rheumatoid Arthritis Patients
}

Isabel M. McFarlane ${ }^{1, *}$, Manjeet S. Bhamra ${ }^{2}$, Abhimnayu Amarnani ${ }^{1}$, Su Yien Zhaz ${ }^{3}$, Srinivas Kolla ${ }^{4}$, Milena Rodriguez Alvarez ${ }^{1}$, George Mo $^{1}$, Maya Srinivasan ${ }^{1}$, Gil Hevroni ${ }^{1}$, Talia Meisel ${ }^{1}$, Abida Hasan ${ }^{1}$, Marie S. Baguidy ${ }^{1}$, Michael Hadaddin ${ }^{1}$, Adielle Melamed ${ }^{1}$, Kristaq Koci $^{1}$, Nicholas Taklalsingh ${ }^{1}$, Joshy Pathiparampil ${ }^{1}$, Latoya Freeman ${ }^{1}$, Ian Kaplan ${ }^{1}$, Naureen Kabani ${ }^{1}$, David J. Ozeri ${ }^{5}$, Elsie Watler ${ }^{1}$, Mosab Frefer $^{1}$, Vytas Vaitkus ${ }^{1}$, Keron Matthew ${ }^{1}$, Fray Arroyo-Mercado ${ }^{1}$, Helen Lyo ${ }^{1}$, Aleksander Feoktistov ${ }^{1}$, Randolph Sanchez ${ }^{6}$, Faisal Soliman ${ }^{7}$, Felix Reyes Valdez ${ }^{8}$, Veena Dronamraju ${ }^{1}$, Michael Trevisonno ${ }^{1}$, Christon Grant ${ }^{1}$, Guerrier Clerger ${ }^{1}$, Kunfeng Sun ${ }^{1}$, Khabbab Amin ${ }^{1}$, Makeda Dawkins ${ }^{1}$, Jason Green ${ }^{1}$, Samir Fahmy ${ }^{3}$, Apoorva Jayarangaiah', Stephen Anthony Waite ${ }^{4}$ and Aaliya Burza ${ }^{10}$

${ }^{1}$ Department of Medicine, Division of Rheumatology SUNY Downstate Medical Center/Health and Hospitals/Kings County, Brooklyn, NY 11203, USA

${ }^{2}$ Department of Medicine, Division of Rheumatology SUNY Upstate Medical University, Syracuse, NY 13210, USA

${ }^{3} \mathrm{NCH}$ Healthcare System Department of Rheumatology, Naples, FL 34110, USA

${ }^{4}$ Department of Radiology SUNY Downstate Medical Center/Health and Hospitals Kings County, Brooklyn, NY 11203, USA

${ }^{5}$ Sheba Medical Center, Tel Aviv 6100000, Israel

${ }^{6}$ Department of Rheumatology, University of Pennsylvania, Philadelphia, PA 19104, USA

${ }^{7}$ Department of Internal Medicine, Baptist Memorial Hospital-Tipton, 1995 Highway 51, South Covington, TN 38019, USA

${ }^{8}$ Department of Pulmonary and Critical Care, Montefiore Medical Center Albert Einstein College of Medicine, Bronx, NY 10468, USA

${ }^{9}$ Department of Internal Medicine, NYC Health and Hospitals/Jacobi Medical Center, Bronx, NY, USA

${ }^{10}$ Department of Medicine, Division of Pulmonary and Critical CareState, SUNY DownstateMedical Center/Healthand Hospitals Kings County, Brooklyn, NY 11203, USA

\section{Abstract}

Background: Rheumatoid arthritis (RA) patients have an increased risk of malignancy with postulated risk factors that include chronic inflammation, smoking and the use of immunosuppressants have been postulated as drivers of higher malignancies rates. Our study aimed to describe the prevalence and type of malignancies encountered in an urban, predominantly Black RA patient population.

Methods: Cross sectional analysis of 1142 patients with RA diagnosis by ICD-codes of which 501 cases met the inclusion criteria for the study. Blacks accounted for $88.4 \%$ of the study population. Fifty-six patients had cancer recorded in their medical records and these cases were further reviewed for tumor type, timing of diagnosis and patient clinical characteristics.

Results: The cancer prevalence was $11.2 \%$ (56/501) in our Black RA population being studied. Mean age at cancer diagnosis was $59.9 \pm 5.2$ for the patients who developed cancer before RA diagnosis and 58.25 \pm 16.02 for those who developed malignancy after RA diagnosis. There were 18 breast cancers, 4 colon and 4 cervical cancers; for lung, multiple myeloma, thyroid, squamous cell carcinoma and pancreas there were 3 cases each; for endometrial, Non-Hodgkin's lymphoma, meningioma and prostate, 2 cases each and 1 each for urinary bladder, esophageal adenocarcinoma, lymphoma, glioblastoma, liver, Hodgkin's lymphoma, sarcoma, ovary and renal cell carcinoma. No differences were found in years of RA duration, joint erosion, joint space narrowing or SENS score except for significantly higher ESR among the cancer group and RF seropositivity in the non-cancer group.

Therapeutic modalities were not significantly different between the cancer and no cancer groups.

Conclusion: Breast cancer was the most prevalent malignancy among our Black RA population. Further studies are needed to identify the contributing factors to the malignancy risk of breast cancer in our Black RA population and whether it is gender-related since RA is more prevalence in women

\section{Publication History:}

Received: March 08, 2020

Accepted: April 23, 2020

Published: April 25, 2020

\section{Keywords:}

Rheumatoid arthritis, Seropositive rheumatoid arthritis, Erosive disease, Breast cancer, Cancer, Solid tumors, Extra-articular manifestations

\section{Key points}

1. First study to assess the prevalence of malignancy in predominantly Black RA patient population.

2. The prevalence of malignancy was $11.2 \%$, with breast cancer being the most frequent followed by colon and cervical cancer.

3. No significant differences were found in patient comorbidities, smoking rates, clinical characteristics, medication patterns between the cancer and non-cancer groups.

\section{Introduction}

Rheumatoid arthritis (RA) is a chronic systemic inflammatory disease characterized by proliferating synovitis, cartilage damage and juxta-articular bone destruction that results in joint deformities and functional disability [1]. In addition, RA has also been associated with significant comorbidities, such as interstitial lung disease, cardiovascular disease, depression and an increased risk of both hematologic and solid malignancies [2,3].
"Corresponding Author: Dr. Isabel M. McFarlane, Department of Internal Medicine, Division of Rheumatology, SUNY-Downstate Medical Center Brooklyn, New York 11203, USA, Tel.: 718-270-2390, Fax: 718-270-1324; E-mail: Isabel.McFarlane@downstate.edu

Citation: McFarlane IM, Bhamra MS, Amarnani A, Zhaz SY, Kolla S, et al. (2020) Prevalence of Malignancy Among Urban Black Rheumatoid Arthritis Patients. Int Clin Res Trials 5: 145. doi: https://doi.org/10.15344/2456-8007/2020/145

Copyright: (c) 2020 McFarlane et al. This is an open-access article distributed under the terms of the Creative Commons Attribution License, which permits unrestricted use, distribution, and reproduction in any medium, provided the original author and source are credited. 
Citation: McFarlane IM, Bhamra MS, Amarnani A, Zhaz SY, Kolla S, et al. (2020) Prevalence of Malignancy Among Urban Black Rheumatoid Arthritis Patients. Int J Clin Res Trials 5: 145. doi: https://doi.org/10.15344/2456-8007/2020/145

Page 2 of 5

While the etiology of RA is multifactorial with a complex interplay of environmental and genetic factors, the pathogenesis is rooted in chronic, systemic inflammatory activation and over activation of the adaptive and innate immune responses [1]. Similarly, inflammation is fundamental to the neoplastic process, whereby inflammatory cells are essential for tumor development, via increased cell proliferation, angiogenesis, evasion of the adaptive immune response, and facilitation of metastasis [4].

The direct link between RA and cancer dates to 1978, when Isomaki established that RA patients had a higher incidence of lymphomas, leukemias, Hodgkin's disease, and myeloma [5]. Since then, numerous follow-up studies including single-center retrospective cohort studies as well as meta-analyses have corroborated Isomaki's findings and expanded on his initial work, showing increased risk of lung cancer and lymphomas and a decreased incidence of colon and breast cancer among patients with RA [6].

Some of the increased risk of malignancy in patients with RA can be linked to common risk factors, with tobacco use lending a common predisposition to lung cancer and RA, and non-steroidal anti-inflammatory drug (NSAID) use, often employed by RA patients for pain control, conferring protection against the development of colon cancer. In the case of RA patients' proclivity to development of lymphomas, it has been hypothesized that the continuous immunologic stimulation results in clonal selection and proliferation of B cells, which are the very culprits of certain lymphomas [6,7]. Given the clear link of immunologic stimulation to development of hematologic malignancies, there is a renewed interest in investigating the link between RA and malignancy.

In recent decades, the development of immunologic and targeted therapies has resulted in slowed disease progression and increased patient survival. While significantly improving disease burden and cause-related mortality, the advent of new therapeutic strategies including disease-modifying anti-rheumatic drugs (DMARDs) raises concern of their impact on concomitant malignancy rates in these patients, given the success of drugs rests on their mechanism of inducing immunosuppression. A recent review article suggested that use of biological DMARDs decrease the overall risk of developing malignancies with the exclusion of hematologic malignancies [8].

Importantly, most of the studies to date which highlight the incidence of malignancy and RA have been based on findings of a patient population wherein the majority are Caucasian, raising the question of the generalizability of these specific cancer type risks to other patient populations.

The goal of the present study is to describe the types of malignancies encountered in an urban, predominantly Black RA patient population.

\section{Methods}

In this cross-sectional, single-center study, we identified patients with RA as either principal or secondary diagnosis, via the International Classification of Diseases, Ninth Revision and Tenth Revision codes (ICD-9-CM 714.0 and ICD-10-CM M06.00-M06.09). All inpatient discharges of patients 18 years or older that took place at the State University of New York Downstate Medical CenterUniversity Hospital of Brooklyn and New York City (NYC) Health + Hospitals/Kings County, from January 1, 2010 to May 30, 2017 were included. Our hospitals serve the predominantly Black population of Central Brooklyn, NY. The protocol was approved by the SUNY Downstate Institutional Review Board [1080808] and the Office of Research Administration at NYC Health + Hospitals/Kings County [001252].

We used physician entries (inpatient/outpatient notes and consultations) and the presence of disease-modifying anti-rheumatic drugs (DMARDs) in the medication reconciliation or DMARDs prescriptions.

Two investigators (IMM, SYZ) independently reviewed the cases identified by ICD codes to confirm RA diagnosis by the 2010 American College of Rheumatology criteria [9].

A standardized study collection data sheet was utilized for data abstraction for the confirmed cases; demographics, clinical data including history of smoking, year of RA-diagnosis, comorbidities, laboratory data, hand imaging and treatment regimens were collected. Cancer diagnosis was confirmed by two investigators (MSB, IMM).

A musculoskeletal radiologist (SK) evaluated the bilateral hand radiographs utilizing the Simple Erosion Narrowing Score (SENS) to ascertain the presence and number of erosions and joint space narrowing [10].

The radiologist was blinded to the serologic status of the cases reviewed. Data are presented as descriptive categorical variables or as mean \pm standard error of the mean $( \pm$ SEM), where applicable. We used t-test to compare between groups for continuous variables, and Chi square analysis for categorical ones. Data analyses were completed via IBM * SPSS version 23.

\section{Results}

Of the 1,142 RA patients identified by ICD codes, 281 had no clinical documentation to support RA diagnosis, 88 had insufficient data for confirmation of RA diagnosis, and 248 had an alternative diagnosis. Forty-eight records were identified as duplicates and abstracted as 24 unique patients. This process resulted in the identification of 501 patients with confirmed RA that were included in this analysis. Women represented $87.8 \%$ of the study cohort. Blacks accounted for $88.4 \%$ and Hispanics $9.2 \%$ of the patient population. Body mass index (BMI) was $28.9 \pm 7.56( \pm$ SD). Mean age was $65.2 \pm 14.4$ for women vs. $61.2 \pm 17$ for men $(\mathrm{p}<0.04)$ (Figure 1$)$.

We encountered a cancer prevalence of $11.2 \%(56 / 501)$ in our RA population. There were 18 breast cancers ( 2 ductal carcinoma), four each for colon and cervical cancer, three each for lung, multiple myeloma, thyroid, squamous cell carcinoma and pancreas; two each for endometrial, Non-Hodgkin's lymphoma, meningioma and prostate and one each for urinary bladder, esophageal adenocarcinoma, lymphoma, glioblastoma, liver, Hodgkin's lymphoma, sarcoma, ovary and renal cell carcinoma. Four patients with usual interstitial pneumonia pattern of RA-related interstitial lung disease (RA-ILD) were found to have developed breast cancer.

In table 1, we present a comparison between the cohorts of RA with cancer and RA without cancer. We did not find significant differences between the cohorts in the clinical parameters such smoking history, body mass index (BMI), traditional cardiovascular (CV) risk factors, $\mathrm{CV}$ outcomes and extra-articular manifestations of RA. In regards to RA-specific characteristics, we found no differences in years of RA 
Citation: McFarlane IM, Bhamra MS, Amarnani A, Zhaz SY, Kolla S, et al. (2020) Prevalence of Malignancy Among Urban Black Rheumatoid Arthritis Patients. Int J Clin Res Trials 5: 145. doi: https://doi.org/10.15344/2456-8007/2020/145

Page 3 of 5

\begin{tabular}{|c|c|c|c|}
\hline & With Cancer & Without Cancer & p value \\
\hline Total Number of Patients & 56 & 445 & \\
\hline Mean Age \pm STD (in years) & $67.4 \pm 11.3$ & $64.3 \pm 15.1$ & NS \\
\hline Number of women (\%) & $87.5 \%$ & $87.8 \%$ & NS \\
\hline \multicolumn{4}{|l|}{ Race/Ethnicity } \\
\hline White & $9.5 \%$ & $69.6 \%$ & NS \\
\hline Black & $88.0 \%$ & $88.3 \%$ & NS \\
\hline Native American & - & $0.2 \%$ & NS \\
\hline Asian Pacific Islander & - & $0.7 \%$ & NS \\
\hline Other & $2.3 \%$ & $3.7 \%$ & NS \\
\hline Hispanics & $8.1 \%$ & $8.3 \%$ & NS \\
\hline \multicolumn{4}{|l|}{ Other Characteristics } \\
\hline Non-smoker & $68.2 \%$ & $70.8 \%$ & NS \\
\hline Ever smoker & $31.8 \%$ & $29.2 \%$ & NS \\
\hline $\mathrm{BMI}($ mean \pm STD $)$ & $28.1 \pm 5.7$ & $29.0 \pm 7.7$ & NS \\
\hline $\mathrm{BMI} \geq 30 \mathrm{Kg} / \mathrm{m}^{2}$ & $27.5 \%$ & $38.4 \%$ & NS \\
\hline $\mathrm{BMI}<20 \mathrm{Kg} / \mathrm{m}^{2}$ & $3.9 \%$ & $8 \%$ & NS \\
\hline Asthma & $14 \%$ & $16.9 \%$ & NS \\
\hline COPD & $17.6 \%$ & $11.1 \%$ & NS \\
\hline Diabetes mellitus & $39.2 \%$ & $27.4 \%$ & NS \\
\hline Hypertension & $75 \%$ & $65.7 \%$ & NS \\
\hline Hyperlipidemia & $45 \%$ & $40.5 \%$ & NS \\
\hline $\mathrm{CAD}$ or $\mathrm{MI}$ & $20.4 \%$ & $19.5 \%$ & NS \\
\hline Cerebrovascular accident & $8.1 \%$ & $9.9 \%$ & NS \\
\hline Chronic kidney disease & $18 \%$ & $11.3 \%$ & NS \\
\hline Venous thromboembolism & $16.3 \%$ & $12.5 \%$ & NS \\
\hline Atrial fibrillation & $12 \%$ & $8 \%$ & NS \\
\hline Congestive heart failure & $19.6 \%$ & $14.7 \%$ & NS \\
\hline At least 1 CVD outcome & $39.3 \%$ & $33.9 \%$ & NS \\
\hline At least 1 traditional CVD risk factor & $90.9 \%$ & $86.9 \%$ & NS \\
\hline$\geq 3$ traditional CVD risk factors & $36.4 \%$ & $32 \%$ & NS \\
\hline Atrial fibrillation & $12 \%$ & $7.9 \%$ & NS \\
\hline Extra-articular manifestations & $8.9 \%$ & $8.1 \%$ & NS \\
\hline Another autoimmune disease & $20 \%$ & $14 \%$ & NS \\
\hline \multicolumn{4}{|l|}{ Rheumatoid Arthritis Characteristics } \\
\hline RA disease duration (years) & $12.2 \pm 9.6$ & $11.8 \pm 8.8$ & NS \\
\hline $\mathrm{RF}+$ & $56.5 \%$ & $77.5 \%$ & $\mathrm{p}<0.05$ \\
\hline $\mathrm{ACPA}+$ & $70.6 \%$ & $69.8 \%$ & NS \\
\hline Either RF + or ACPA + & $89.5 \%$ & $86.7 \%$ & NS \\
\hline Pos RF and Pos ACPA & $58.8 \%$ & $53.2 \%$ & NS \\
\hline ANA positive & $47.1 \%$ & $39.4 \%$ & NS \\
\hline $\mathrm{CRP}>10^{*}$ & $71.4 \%$ & $62.5 \%$ & NS \\
\hline ESR rate $>42^{* *}$ & $81.5 \%$ & $61.8 \%$ & $\mathrm{p}<0.05$ \\
\hline Abnormal hand radiographs & $95.2 \%$ & $94.7 \%$ & NS \\
\hline Joint space narrowing & $61.9 \%$ & $69.9 \%$ & NS \\
\hline Presence of Joint erosions & $66.6 \%$ & $66.8 \%$ & NS \\
\hline SENS $( \pm$ SD) & $25.4 \pm 18.5$ & $31.0 \pm 21.7$ & NS \\
\hline Steroids only & $51.1 \%$ & $56.6 \%$ & NS \\
\hline Methotrexate & $31.9 \%$ & $41.3 \%$ & NS \\
\hline Other DMARD & $52.6 \%$ & $41.9 \%$ & NS \\
\hline Only Biologics & $14.3 \%$ & $16.5 \%$ & NS \\
\hline Steroids + any DMARD/Biologics & $30.3 \%$ & $36.2 \%$ & NS \\
\hline Only DMARD/Biologics & $30.3 \%$ & $26.3 \%$ & NS \\
\hline
\end{tabular}

Table 1: RA Clinical Characteristics for Cancer and No Cancer Cohorts.

Percentages were calculated based on the number of patients with data available on the measure. ACPA: anti-citrullinated peptide antibody, BMI: body mass index, CAD: coronary artery disease, COPD: chronic obstructive pulmonary disease, CRP: C reactive protein, CVD: cardiovascular disease, DMARD: disease modifying anti-rheumatic drugs, ESR: erythrocyte sedimentation rate, MI: myocardial infarction, RF: rheumatoid factor, SENS: simple erosion narrowing score.

${ }^{* *} \mathrm{C}$ reactive protein $>10 \mathrm{mg} / \mathrm{L}$. is associated with MI [11].

${ }^{* * *}$ Erythrocyte sedimentation rate $>42 \mathrm{~mm} / \mathrm{hr}$. is associated with $\mathrm{MI}$ and ischemic stroke risk $[11,12]$.

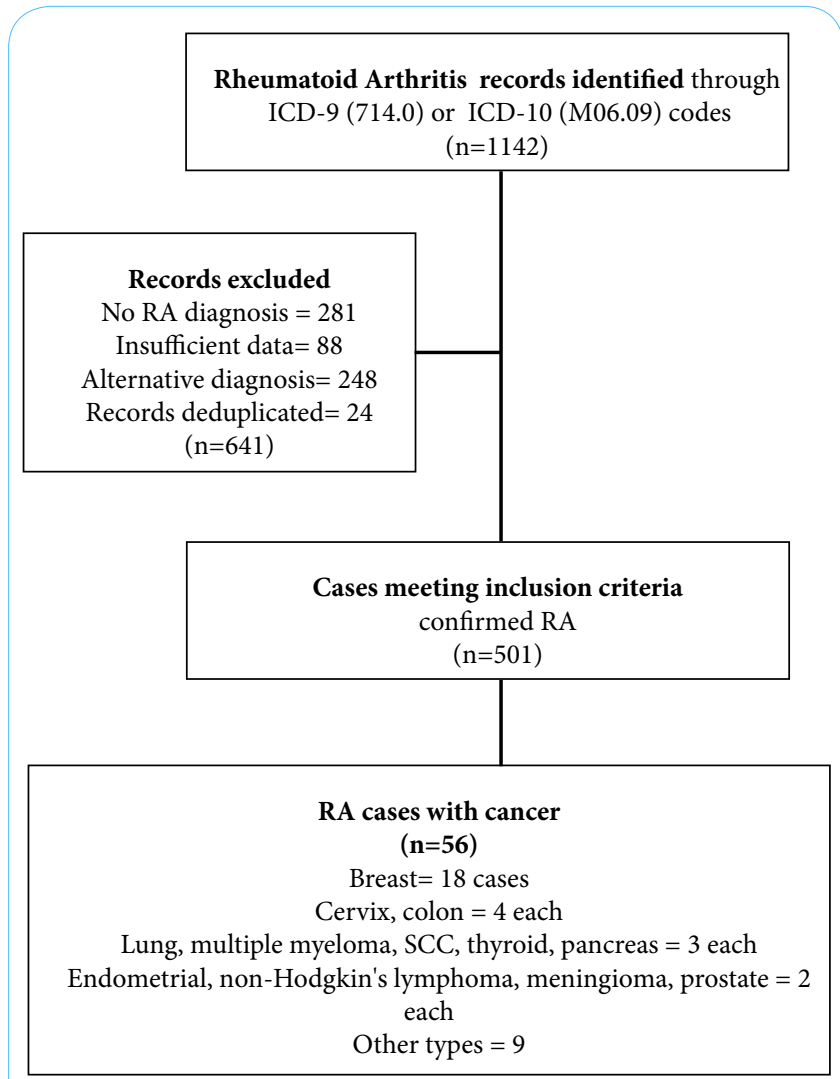

Figure 1: Flow chart showing the selection procedure of rheumatoid arthritis cases in this study.

disease, joint erosion, joint space narrowing or SENS score except for significantly higher ESR among the cancer group and RF seropositivity in the non-cancer group. Therapeutic modalities for the two groups were also examined and exposure to corticosteroids, DMARDS and biologics revealed no significant differences.

We also assessed the sequence of cancer diagnosis to RA diagnosis for the 22 cases with available data. Twelve patients had developed cancer after RA diagnosis (mean, 10.75 years; range 5-22 years), 8 had developed cancer before RA diagnosis (mean, 6.75 years; range 3-11 years) and 2 patients received the two diagnosis the same year. Among the patients who had developed cancer after RA diagnosis, $66 \%$ had RA-specific risk factors however, no differences were found in regards to exposure to RA therapies prior to malignancy diagnosis.

The mean age at cancer diagnosis was $59.9 \pm 5.2$ for the patients who developed cancer before RA diagnosis which was similar to that of the patients (58.25 \pm 16.02$)$ who developed malignancy after RA diagnosis.

In regards to overall survival for the cancer cohort, $43.4 \%$ of the patients were alive, $32.1 \%$ of the patients were lost to follow-up and $24.5 \%$ had expired by the date of completion of the study.

A sub-analysis of the breast cancer patients showed that 11 were alive $(61 \%), 3$ had expired (16.6\%) and 4 patients $(22.2 \%)$ had been lost to follow up.

\section{Discussion}

The prevalence rate of cancer encountered in this predominantly Black RA population was $11.2 \%$. The incidence of malignancy in RA in a recently published study from Beijing was $4.16 \%$. Additionally, 
Citation: McFarlane IM, Bhamra MS, Amarnani A, Zhaz SY, Kolla S, et al. (2020) Prevalence of Malignancy Among Urban Black Rheumatoid Arthritis Patients. Int J Clin Res Trials 5: 145. doi: https://doi.org/10.15344/2456-8007/2020/145

a prospective study conducted in Olmsted County, MN, showed a $23.8 \%$ incidence of malignancy among RA Caucasian patients $[13,14]$.

The association of RA and malignancy was recognized in the late 70's in an important study conducted on Caucasian RA patients with the number of malignancies such as leukemia, lymphomas, Hodgkin's disease and myeloma were found to be more than double of the expected for the general population [5].

The association between RA and malignancies was again demonstrated in a meta-analysis based on published data on a Swedish RA population. The risk of lymphoma and lung cancer were noted to be higher for the RA patients while breast and colorectal cancers were lower among the RA cohort. Risk factors such as cigarette smoking, chronic lung inflammation, and presence of interstitial lung disease in RA patients were hypothesized to play a role in the development of cancer [15].

Published studies focusing on Caucasian RA population consistently reported the increased risk for lymphoma and lung cancer while cervical, prostate cancer and melanoma were seen with a frequency similar to that general population [16].

Another study on a Caucasian population reported a small to moderately increased risk of malignancies in the range of $5-10 \%$ among RA patients if non-melanoma skin cancers were excluded; the risk was highest for hematologic malignancies. Lung cancer risk appeared to be also increased. The risk of malignancy in RA did not appear to be related to the use of DMARDs or biologics such as TNF inhibitors [13].

When long standing and recent onset RA patients were compared in a large Caucasian cohort the risk of lymphoproliferative disease or solid tumor was not elevated $[7,17,18]$.

Our population demonstrated a higher frequency of cancer than previously described among Caucasian cohorts and breast cancer was seen in $32.14 \%$ of the cases in contrast with $23 \%$ (17/74) found in the Beijing study. Conflicting reports exists in regards to breast cancer and its association with RA. One study reported breast cancer to be lower in RA patients than in the general population however, Blacks made up only 7\% of the entire patient cohort [19]. On the other hand, increased risk of breast cancer in non-Caucasian RA patients was recognized in a meta-analysis [20].

The incidence trends of all site cancers and lymphoma, in Veteran RA population, showed a significant decrease with the introduction of biologics comparing 2001-2005 to 2006-2010. The population was predominantly Caucasian (80\%) and might suffer from potential referral bias [21].

Our study demonstrated significantly higher ESR values among the RA group with cancer than without cancer $(p<0.05)$. The association between inflammation and malignancy has been well-described, whereby persistent immunologic stimulation and the presence of inflammatory mediators leads to genetic instability and accumulation of random genetic alterations in cancer cells [13]. Further, high frequencies of p53 mutations similar to those found in tumor cells have been described in RA [15]. Additionally, immunostimulation, rather than immunosuppression mediated by RA treatments, has been considered the major driving force in the increased incidence of inflammation-associated lymphomas seen in RA [17,22].

The BMI between the two patient groups was not significantly different. However, there was a higher rate of obesity $($ BMI $>30)$ among those with cancer consistent with the notion that obesity is associated with increased cancer risk.

Further, we observed significantly different rates in RF seropositivity between patients with and without cancer which likely express disease severity however, no difference was found in the frequency of DMARDs or biologics use between the groups.

The fact that we encountered a cancer prevalence of $11.2 \%$ among our RA population could be explained by the heightened

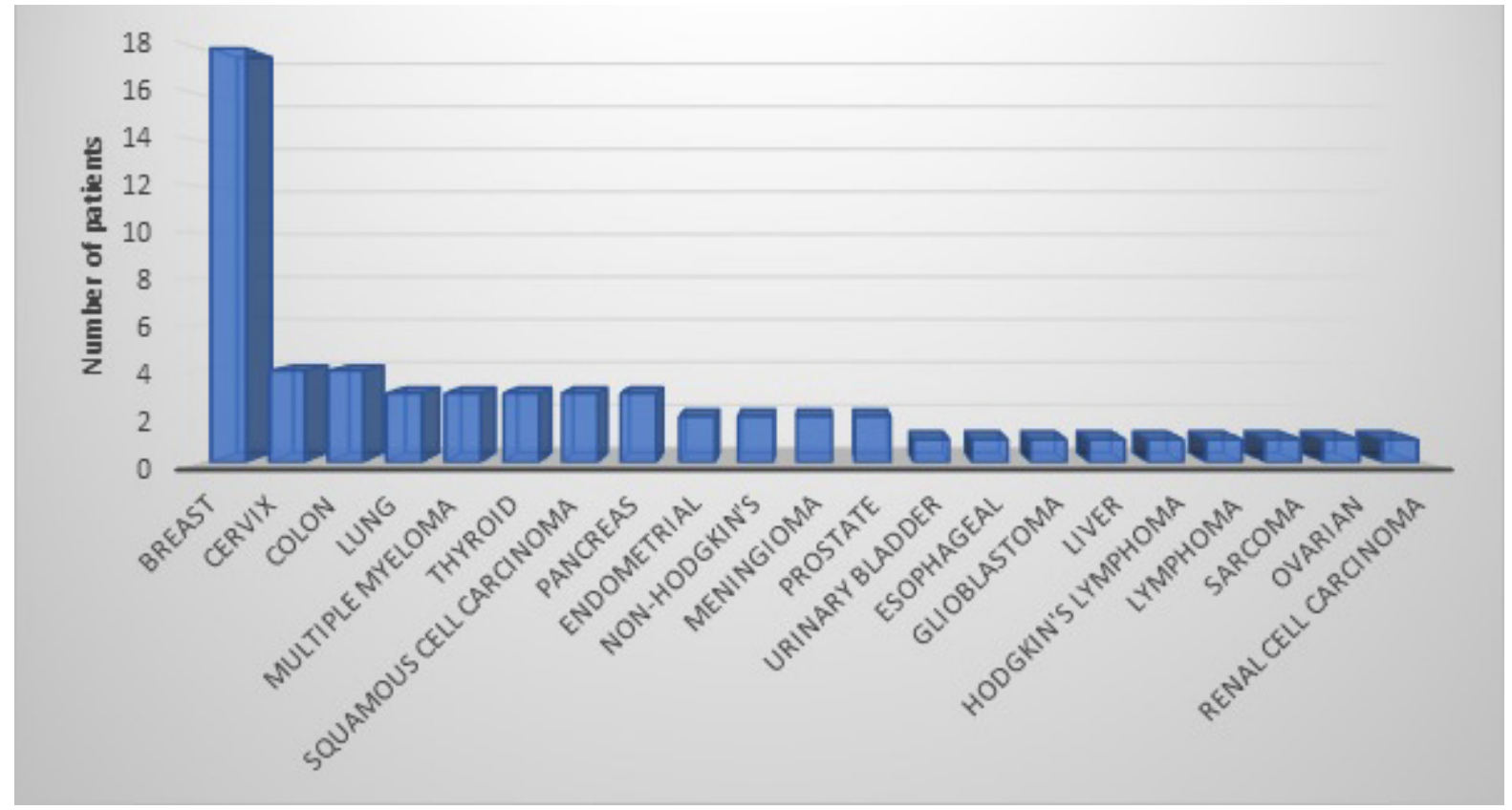

Figure 2: Types of Malignancies in 56 RA patients. 
Citation: McFarlane IM, Bhamra MS, Amarnani A, Zhaz SY, Kolla S, et al. (2020) Prevalence of Malignancy Among Urban Black Rheumatoid Arthritis Patients. Int J Clin Res Trials 5: 145. doi: https://doi.org/10.15344/2456-8007/2020/145

Page 5 of 5

surveillance provided to RA patients that allowed for early detection of malignancies given the use of DMARDs as therapeutic agents that are known to confer an increased risk of malignancy (Figure 2).

Finally, our study is limited by small sample size, the retrospective nature of the analysis, lack of RA-specific disease activity measurements, anatomic pathology characterization, and staging of the cancers, response to therapeutic interventions, changes made to RA therapy and survival outcomes. Inaccuracy in coding explains the number of cases in which RA diagnosis was not found in the clinical documentation. There is also data missing at random which we believe is harmless and does not represent a systematic bias.

\section{Conclusions}

This is the first study to evaluate malignancy in our predominantly Black RA population with a prevalence of $11.2 \%$.

Breast cancer was the most prevalent malignancy among our Black RA population, which is consistent with previously published report that breast cancer risk is increased among patients with RA [20]. Cancer patients with RA were found to have a significantly higher ESR than non-cancer patients, likely reflecting higher levels of inflammation due to the dual disease process.

No significant differences were found in patient comorbidities, smoking rates, clinical characteristics, medication patterns between the cancer and non-cancer groups.

Further studies are needed to understand the higher overall risk of malignancy among our Black RA population. Possible risk factors include higher rates of obesity, smoking, advanced age, use of steroid and immunosuppressive drugs, as well as high levels of inflammatory markers among our patients.

\section{Funding}

This work is supported in part by Dr. Moro O. Salifu's efforts through NIH Grant \# S21MD012474.

\section{Acknowledgments}

We are grateful to Chao Ma, Sharlene Mills, Denton Smith, Stuart Clenman for their help with data retrieval and organization.

\section{Competing Interests}

The authors declare that they have no competing interests.

\section{References}

1. McInnes IB, Schett $G$ (2011) The pathogenesis of rheumatoid arthritis. $N$ Engl J Med 365: 2205-2219.

2. Baillet A, Gossec L, Carmona L, Wit M, Eijk-Hustings $Y$ et al. (2016) Points to consider for reporting, screening for and preventing selected comorbidities in chronic inflammatory rheumatic diseases in daily practice: a EULAR initiative. Ann Rheum Dis 75: 965-973.

3. Dougados $M$, Soubrier $M$, Antunez A, Balint $P$, Balsa A, et al. (2014) Prevalence of comorbidities in rheumatoid arthritis and evaluation of their monitoring: results of an international, cross-sectional study (COMORA) Ann Rheum Dis 73: 62-68.

4. Colotta F, Allavena P, Sica A, Garlanda C, Mantovani A, et al. (2009) Cancerrelated inflammation, the seventh hallmark of cancer: links to genetic instability. Carcinogenesis 30: 1073-1081.

5. Isomäki $\mathrm{H}$, Hakulinen $\mathrm{T}$, Joutsenlahti $U$ (1978) Excess risk of lymphomas, leukemia and myeloma in patients with rheumatoid arthritis. J Chronic Dis 31: 691-696.
6. De Cock D, Hyrich K (2018) Malignancy and rheumatoid arthritis: Epidemiology, risk factors and management. Best Pract Res Clin Rheumatol 32: 869-886.

7. Smitten AL, Simon TA, Hochberg MC, Suissa S (2008) A meta-analysis of the incidence of malignancy in adult patients with rheumatoid arthritis. Arthritis Res Ther 10: 45.

8. Cho SK, Lee J, Han M, Bae SC, Sung YK, et al. (2017) The risk of malignancy and its incidence in early rheumatoid arthritis patients treated with biologic DMARDs. Arthritis Res Ther 19: 277.

9. Aletaha D, Neogi T, Silman AJ, Funovits J, Felson DT, et al. (2010) 2010 rheumatoid arthritis classification criteria: an American College of Rheumatology/European League Against Rheumatism collaborative initiative. Ann Rheum Dis 62: 2569-2581.

10. Dias EM, Lukas C, Landewé R, Fatenejad S, van der Heijde D, et al. (2008) Reliability and sensitivity to change of the Simple Erosion Narrowing Score compared with the Sharp-van der Heijde method for scoring radiographs in rheumatoid arthritis. Ann Rheum Dis 67: 375-379.

11. Zhang J, Chen L, Delzell E, Muntner P, Hillegass WB, et al. (2014) Republished: The association between inflammatory markers, serum lipids and the risk of cardiovascular events in patients with rheumatoid arthritis. Postgrad Med J 90: 722-729.

12. Myasoedova E, Crowson CS, Kremers HM, Roger VL, Fitz-Gibbon PD, et al. (2011) Lipid paradox in rheumatoid arthritis: the impact of serum lipid measures and systemic inflammation on the risk of cardiovascular disease. Ann Rheum Dis 70: 482-487.

13. Raheel S, Crowson CS, Wright K, Matteson EL, et al. (2016) Risk of malignant neoplasm in patients with incident rheumatoid arthritis 1980-2007 in relation to a comparator cohort: a population-based study. Int J Rheumatol 2016: 4609486.

14. Wang $\mathrm{YH}$, Zhang GH, Zhang LL, Luo JL, Gao L, et al. (2018) Clinical characteristic of 74 cases of malignant tumor in rheumatoid arthritis. Beijing Da Xue Xue Bao Yi Xue Ban 50: 986-990.

15. Smitten AL, Simon TA, Hochberg MC, Suissa $S$, et al. (2008) A meta-analysis of the incidence of malignancy in adult patients with rheumatoid arthritis. Arthritis Res Ther 10: 45 .

16. Simon TA, Thompson A, Gandhi KK, Hochberg MC, Suissa S, et al. (2015) Incidence of malignancy in adult patients with rheumatoid arthritis: a metaanalysis. Arthritis Res Ther 17: 212

17. Andersen $\mathrm{CL}$, Lindegaard $\mathrm{H}$, Vestergaard $\mathrm{H}$, Siersma VD, Hasselbalch $\mathrm{HC}$ et al. (2014) Risk of lymphoma and solid cancer among patients with rheumatoid arthritis in a primary care setting. PLoS One 9: 99388.

18. Smolen JS, Landewé R, Bijlsma J, Burmester G, Chatzidionysiou K, et al. (2017) EULAR recommendations for the management of rheumatoid arthritis with synthetic and biological disease-modifying antirheumatic drugs: 2016 update. Ann Rheum Dis 76: 960-977.

19. Parikh-Patel A, White RH, Allen M, Cress R et al. (2009) Risk of cancer among rheumatoid arthritis patients in California. Cancer Causes Control 20: 1001-1010.

20. Tian G, Liang JN, Wang ZY, Zhou D, et al. (2014) Breast cancer risk in rheumatoid arthritis: an update meta-analysis. Biomed Res Int 2014: 453012.

21. Singh N, Gao Y, England BR, Rould P, Field E, et al. (2018) All Site Cancers and Lymphoma Incidence in US Veterans with Rheumatoid Arthritis 20012015. Arthritis Rheumatol.

22. Baecklund E, Sundström C, Ekbom A, Catrina AI, Biberfeld P, et al. (2003) Lymphoma subtypes in patients with rheumatoid arthritis: increased proportion of diffuse large B cell lymphoma. Arthritis Rheum 48: 1543-1550. 